\title{
Papers
}

\section{Re-engineering pathology: an IT solution to the data conundrum}

\author{
M D Simmons, J Nadin, $M$ Isaac
}

\begin{abstract}
Aims-To develop a simple process for data transfer between disparate laboratory computer systems, and to assist in the rationalisation of services while maintaining or improving clinical care.

Methods-Using existing hardware and software in the laboratories, data manipulation was reduced in the sending and receiving laboratories by using scripting languages to perform the import and export routines from disparate laboratory systems.

Results-Data exchange between Carmarthen and Swansea laboratories (12 000 specimens per year) was achieved in a semiautomatic manner for most specimens. Turnaround times were equalled or improved with the new arrangements, and significant reductions were obtained in staff costs.

Conclusions-Data exchange between disparate laboratory computer systems can be achieved inhouse using simple systems. The process offers a route to assist in the management of reducing resources. The process could be readily utilised in other pathology disciplines or with any data integration exercise. There is scope for further enhancement that is currently being investigated.

(F Clin Pathol 1997;50:815-818)
\end{abstract}

Keywords: information management; laboratory rationalisation; computing

Public Health

Laboratory,

Carmarthen, UK

M D Simmons

J Nadin

Public Health

Laboratory, Swansea,

UK

M Isaac

Correspondence to:

Dr Simmons, Public Health

Laboratory, West Wales

General Hospital,

Carmarthen SA31 2AF, UK.

Accepted for publication 15 July 1997 between laboratories. This process has made major contribution to reducing turnaround times, while reducing costs.

\section{Background}

The PHLS, in common with all areas of the NHS and public sector, is expected to make continuous improvements in its use of resources. The PHLS commissioned an independent strategic review ${ }^{1}$ to advise on the most appropriate way to accommodate this process.

The major change accepted by the PHLS from the review committee was for the reorganisation of the PHLS into a series of groups that would allow laboratories to work together to improves services and reduce costs. PHLS Wales was formed in April 1995 as one of the two pioneer group laboratories.

A series of working groups looked at all aspects of the service in Wales. Work patterns and staff mix were reviewed, and early consideration given to changing the pattern of virology service delivery. Such rationalisation was seen as being compatible with the recommendations of the Strategic review of pathology. ${ }^{2}$

West Wales locality rationalisation

The Carmarthen Laboratory receives some 12000 virology and serology requests per year. When undertaken on site, work is batched on a weekly basis for most tests apart from those undertaken on an urgent basis (such as hepatitis serology, antigen detection from respiratory secretions, and Clostridium difficille toxin tests). It was recognised that the potential existed for improving specimen turnaround times if work was transferred to the larger laboratory at Swansea (30 miles away) where serological tests are undertaken on a more frequent basis. To achieve this objective, efficient specimen transport was necessary together with a means of integrating laboratory computer systems to facilitate the transfer of specimen details and results. The latter was also important to ensure that any reduction in staff costs were maximised by avoiding any increase in clerical activity associated with this initiative. The Carmarthen-Swansea link was implemented in November 1995 as a pilot in advance of further links between public health laboratories in Wales being established.

\section{Laboratory computer integration}

The West Wales locality provided a challenging situation for computer integration because of 


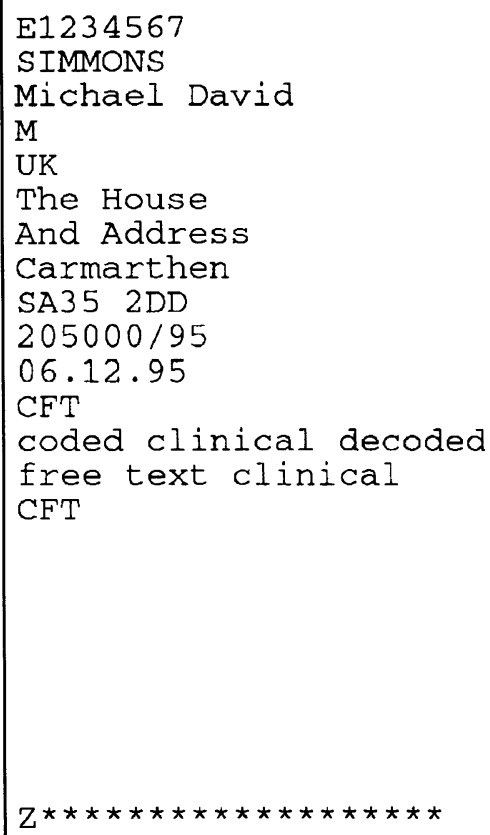

Figure 1 Single request exported from Telepath.

the diversity of the systems. The Swansea laboratory computer uses the ACT CILMS system running on a VAX mainframe computer. Carmarthen has a CDS Telepath system running on an IBM RS6000 mini-computer.

The goal to be achieved was single data entry. Carmarthen would be responsible for request entry with an automatic upload to the Swansea system. Swansea would be responsible for input of results with automatic upload into the Carmarthen system. An early decision was taken to assign a unique block of laboratory numbers to the Carmarthen specimens that would be used in the Swansea system. We also established that hospital numbers used a different format at the two sites. In addition, computer generated hospital numbers were different in the two systems. Thus, the unique common identifier was the laboratory number. Where a hospital number was generated by the Carmarthen system, this would be replicated in the Swansea system. Four separate operations were therefore required, these are described below.

EXPORT FROM TELEPATH

To export data electronically from Telepath, a fixed length ASCII text file is produced by the system. The report generator within Telepath is used to create the necessary report format. This requires the creation of a report with fields only, each occupying a new line of the report. There is no additional text in the report, other than that of an end of record marker at the bottom of the report. A typical copy of a single request is illustrated in fig 1 .

The Carmarthen Telepath system had to be instructed to produce a report as a text file for export to Swansea. This is achieved by forcing an interim report to be issued. The report sim- ply reads "specimen in progress". The Telepath logic trees ("rule base processing") are instructed to examine for the code for "specimen in progress" and if found to issue a non-printing report to the requester, and a copy report to Swansea.

Specimens are entered during the day until 1500 when the courier picks up the daily batch of specimens for Swansea. At this stage, the clerical staff do a "print run" of the Swansea specimens to a UNIX text file. This is transferred to the DOS environment using File Transfer Protocol (FTP) and transmitted to Swansea by EpiNet. Details of specimens due from Carmarthen are therefore available in Swansea about an hour in advance of the specimens arriving. In practice, any email system could be used or a floppy disk could be sent with the specimens. If an open email system were to be used, data encryption should be considered.

\section{IMPORT INTO CILMS}

Data from Carmarthen arrives in Swansea via EpiNet. The file is read into the CILMS system using a script program written for Procomm Plus (Datastorm). Procomm Plus is a communication utility program that allows access to a variety of platforms from a personal computer. The script file is used to mirror keystrokes during data entry into the CILMS system, taking data items from the file exported from Telepath. The script file branches as necessary to take account of whether the patient has previously been entered into the system. Clinical data is entered as free text.

EXPORT OF RESULTS FROM CILMS

When the work has been done, results are printed as normal. The results must also be made available in electronic format for export to Carmarthen. A standard export routine is not available as part of the Swansea CILMS system. As an interim solution therefore, screen capture of a repeat print run was considered the most appropriate method of data export. Screen capture is achieved using Cross Talk. The captured text file has a standard format albeit complete with characters that represent printer formatting instructions. The screen captured text files are transmitted to Carmarthen via EpiNet.

\section{IMPORT OF RESULTS TO TELEPATH}

The files as received contain expanded textural information rather than codes. Each record is of a fixed length and individual data items are contained in consistent blocks. Each file is therefore read by a program result by result. This program was written within a standard database package (Open Access IV, Software Products International).

As laboratory numbers are duplicated by both systems, only a limited amount of data is required from each result. The textural result is picked out and compared with information 


\begin{tabular}{|c|}
\hline 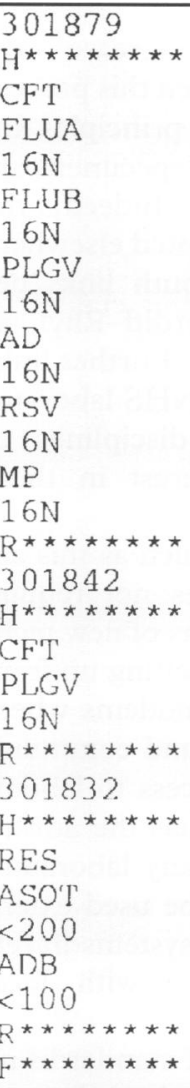 \\
\hline
\end{tabular}

Figure 2 Data result for import into Telepath.
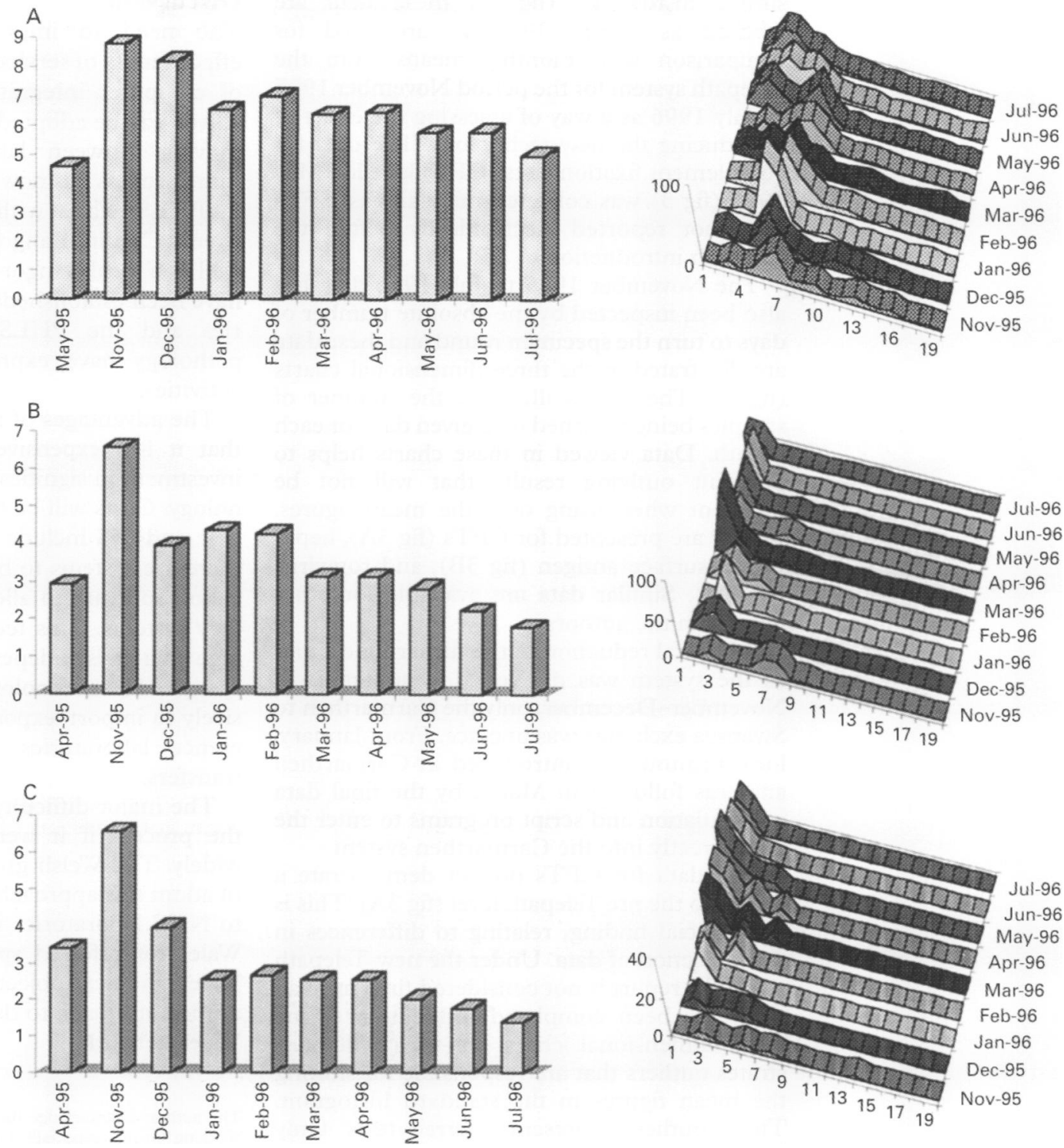

Figure 3 Left, histograms showing the number of days to turnaround a specimen. Right, three dimensional charts showing the number of samples being returned on a given day for each month. (A) complement fixations tests; $(B)$ hepatitis $B$ surface antigen; $(C)$ rotavirus.

stored in the database. Where the organism and result is found, the appropriate Telepath code is collected from the database. The extracted information is used to create a new ASCII text file that will contain coded results for entry into the Telepath system. In addition, as the file received from Swansea is read, a copy is printed locally in Carmarthen together with an assessment of whether the computer will attempt to enter the result. Error trapping is built into the program to check that the result is entirely contained on one electronic page, that the organisms and results are known to the database, and that there is nothing new about the result type. If there is any apparent anomaly, the printed report is flagged with a message to enter the results manually. A copy of the variable length result file for import into Telepath is presented in fig 2 .

Once the file has been read and the code generated, the PC loads Novix Host Presenter (Firefox) in the Microsoft Windows environment and runs a script to take the data into
Telepath. The hard copy printed results are attached to the original request form and given to the authoriser. Any results that the computer has flagged for manual entry go to a member of the clerical staff for entry before being passed for authorisation. As data entry is included in the requesting laboratory computer system, the normal validation processes used for inhouse tests can be applied to tests referred off site.

During the process extensive use is made of menu driven options on the PC that enable clerical and scientific staff to undertake the transfers without detailed knowledge of the processes involved.

\section{Results}

Approximately $95 \%$ of results were entered automatically by the computer. Turnaround times were used to assess the impact of this technology. During April 1995, before the introduction of the Telepath system for virology, turnaround time data were collected using the previous inhouse computer system. In the 
simple histograms (fig 3), these data are labelled as April 1995 and are used for comparison with monthly means from the Telepath system for the period November 1995 to July 1996 as a way of assessing the effect of introducing the new technology. The data for complement fixation tests (CFT) labelled May 1995 (fig 3) was collected manually as CFTs were not reported electronically before the Telepath introduction.

The November 1995 to July 1996 data has also been inspected by the absolute number of days to turn the specimen round and these data are illustrated in the three dimensional charts (fig 3). The charts illustrate the number of samples being returned on a given day, for each month. Data viewed in these charts helps to pinpoint outlying results that will not be apparent when using only the mean figures. Charts are presented for CFTs (fig 3A), hepatitis $B$ surface antigen (fig $3 B$ ), and rotavirus (fig 3C). Similar data are available for other tests from the authors.

A general reduction in the turnaround times as the system was developed is apparent. In November-December, only the Carmarthen to Swansea exchange was in place. From January, local printout was introduced in Carmarthen and was followed in March by the final data manipulation and script programs to enter the data directly into the Carmarthen system.

The data for CFTs do not demonstrate a return to the pre Telepath level (fig $3 \mathrm{~A}$ ). This is an artificial finding, relating to differences in the collection of data. Under the new Telepath system, a report is not considered final until all work has been completed. Inspection of the three dimensional chart for CFTs demonstrates outliers that are responsible for shifting the mean figures in the standard histogram. These outliers represent referred tests. Only inhouse tests were included in the CFT calculations for the May 1995 data.

In addition to maintaining and in many cases improving specimen turnaround times in the Carmarthen laboratory, this rationalisation process has led to a significant reduction in staff costs.

\section{Discussion}

The need to improve and increase cost effectiveness of services has driven this process of computer integration. The principles involved can be adopted wherever specimens are moving between laboratories. Indeed, the same process has now been adopted elsewhere in PHLS Wales with north-south links between Cardiff-Bangor and Cardiff-Rhyl, as well as a local Bangor-Rhyl link. Further links have been established between NHS laboratories and the PHLS. Other disciplines of pathology have expressed interest in these activities.

The advantages of a system such as this are that it is inexpensive and does not require investment in significant amounts of new technology. Costs will be limited if setting up from new and will include costs of modems where there are systems to be linked, and communication software to allow PC access to laboratory systems. This technology has the advantage that it is independent of any laboratory system. The technology could be used extensively to import-export data to systems in reference laboratories as well as with local transfers.

The major difficulty is one of standardising the process if it were to be adopted more widely. The Welsh group is already beginning to adopt this approach in delivering its services to NHS laboratories in Wales that use PHLS Wales for referred specimens. Within PHLS Wales, the next objective is to develop a more unified approach to data sharing with a PHLS Wales network.

The authors acknowledge the support of the PHLS Wales IT Working Group, especially Dr R Henry, Computer Manager, CDSC (Welsh Unit) who supplied the initial script program that was amended and used for the first data import routine.

1 Public Health Laboratory Service. Report of a strategic review of the Public Health Laboratory Service. London: PHLS, 1994

2 NHS Executive. Strategic review of pathology services. The development of the service. London: HMSO 1995:chapter 4. 